\title{
Dose of Prophylactic Platelet Transfusions and Prevention of
}

\section{Hemorrhage}

\begin{abstract}
Sherrill J. Slichter, M.D., Richard M. Kaufman, M.D., Susan F. Assmann, Ph.D., Jeffrey McCullough, M.D., Darrell J. Triulzi, M.D., Ronald G. Strauss, M.D., Terry B. Gernsheimer, M.D., Paul M. Ness, M.D., Mark E. Brecher, M.D., Cassandra D. Josephson, M.D., Barbara A. Konkle, M.D., Robert D. Woodson, M.D., Thomas L. Ortel, M.D., Ph.D., Christopher D. Hillyer, M.D., Donna L. Skerrett, M.D., Keith R. McCrae, M.D., Steven R. Sloan, M.D., Ph.D., Lynne Uhl, M.D., James N. George, M.D., Victor M. Aquino, M.D., Catherine S. Manno, M.D., Janice G. McFarland, M.D., John R. Hess, M.D., Cindy Leissinger, M.D., and Suzanne Granger, M.S. From the Puget Sound Blood Center and University of Washington Medical Center, Seattle (S.J.S., T.B.G.); Brigham and Women's Hospital (R.M.K.), Children's Hospital Boston (S.R.S.), and Beth Israel Deaconess Medical Center (L.U.) - all in Boston; New England Research Institutes, Watertown, MA (S.F.A., S.G.); Fairview-University Medical Center, Minneapolis (J.M.); University of Pittsburgh, Pittsburgh (D.J.T.); University of lowa, lowa City (R.G.S.); Johns Hopkins University (P.M.N.) and University of Maryland ( J.R.H.) - both in Baltimore; University of North Carolina, Chapel Hill (M.E.B.); Emory University, Atlanta (C.D.J., C.D.H.); University of Pennsylvania (B.A.K.) and Children's Hospital of Philadelphia (C.S.M.) - both in Philadelphia; Blood Center of Wisconsin, Milwaukee, and University of Wisconsin-Madison, Madison (R.D.W., J.G.M.); Duke University, Durham, NC (T.L.O.); Weill College of Cornell University, New York (D.L.S.); Case Western Reserve University, Cleveland (K.R.M.); University of Oklahoma, Oklahoma City ( J.N.G.); University of Texas Southwestern Medical Center, Dallas (V.M.A.); and Tulane University Hospital and Clinics, New Orleans (C.L.).
\end{abstract}

\section{Abstract}

BACKGROUND-We conducted a trial of prophylactic platelet transfusions to evaluate the effect of platelet dose on bleeding in patients with hypoproliferative thrombocytopenia.

\begin{abstract}
METHODS-We randomly assigned hospitalized patients undergoing hematopoietic stem-cell transplantation or chemotherapy for hematologic cancers or solid tumors to receive prophylactic platelet transfusions at a low dose, a medium dose, or a high dose $\left(1.1 \times 10^{11}, 2.2 \times 10^{11}\right.$, or $4.4 \times 10^{11}$ platelets per square meter of body-surface area, respectively), when morning platelet counts were 10,000 per cubic millimeter or lower. Clinical signs of bleeding were assessed daily. The primary end point was bleeding of grade 2 or higher (as defined on the basis of World Health Organization criteria).
\end{abstract}

\begin{abstract}
RESULTS-In the 1272 patients who received at least one platelet transfusion, the primary end point was observed in $71 \%, 69 \%$, and $70 \%$ of the patients in the low-dose group, the medium-dose group, and the high-dose group, respectively (differences were not significant). The incidences of higher grades of bleeding, and other adverse events, were similar among the three groups. The median number of platelets transfused was significantly lower in the low-dose group $\left(9.25 \times 10^{11}\right)$ than in the medium-dose group $\left(11.25 \times 10^{11}\right)$ or the high-dose group $\left(19.63 \times 10^{11}\right)(\mathrm{P}=0.002$ for low vs. medium, $\mathrm{P}<0.001$ for high vs. low and high vs. medium), but the median number of platelet transfusions given was significantly higher in the low-dose group (five, vs. three in the medium-dose
\end{abstract}

Copyright (C) 2010 Massachusetts Medical Society.

Address reprint requests to Dr. Slichter at Puget Sound Blood Center, 921 Terry Ave., Seattle, WA 98104-1256, or at sjslichter@ psbc.org. 
and three in the high-dose group; $\mathrm{P}<0.001$ for low vs. medium and low vs. high). Bleeding occurred on $25 \%$ of the study days on which morning platelet counts were 5000 per cubic millimeter or lower, as compared with $17 \%$ of study days on which platelet counts were 6000 to 80,000 per cubic millimeter $(\mathrm{P}<0.001)$.

CONCLUSIONS-Low doses of platelets administered as a prophylactic transfusion led to a decreased number of platelets transfused per patient but an increased number of transfusions given. At doses between $1.1 \times 10^{11}$ and $4.4 \times 10^{11}$ platelets per square meter, the number of platelets in the prophylactic transfusion had no effect on the incidence of bleeding. (ClinicalTrials.gov number, NCT00128713.)

The optimal number of platelets in a prophylactic platelet transfusion is controversial. ${ }^{1,2} \mathrm{~A}$ standard dose for adults is considered to be approximately $3 \times 10^{11}$ to $6 \times 10^{11}$ platelets. ${ }^{3}$ Higher doses than these could potentially result in superior hemostasis, ${ }^{4}$ but a lower dose might be equally effective while conserving the platelet supply. Two randomized trials with limited enrollment - one of 111 patients ${ }^{5}$ and the other of 119 patients $^{6}$ - have compared a low dose of platelets to the standard dose. In both trials, the two doses prevented bleeding to a similar degree. We conducted a randomized trial of prophylactic platelet transfusions to determine the effects of the dose of platelets on clinical signs of bleeding, the use of platelet and red-cell transfusions, changes in the recipient's post-transfusion platelet count, days to next transfusion, and adverse events. ${ }^{7}$

\section{METHODS}

A subcommittee of the Transfusion Medicine/Hemostasis Clinical Trials Network investigators designed the study and wrote the manuscript, which was reviewed by all authors. Site coordinators gathered the data and submitted the results electronically. (The study investigators and staff are listed in the Appendix.) The data were analyzed with the use of SAS software (version 9.2). ${ }^{8}$ The lead author wrote the first draft of the manuscript and vouches for the completeness and accuracy of the data. Institutional review boards approved the study. Adults provided written informed consent; and for children, a parent or legal guardian provided written informed consent. Children provided assent if required by local site policy. A data and safety monitoring board reviewed the data twice a year. Stopping boundaries for comparison of the primary end point between each pair of treatment groups were calculated with the use of an alpha spending function similar to O'Brien-Fleming boundaries.

\section{ELIGIBILITY CRITERIA}

Patients were eligible for the study if they were inpatients of any age undergoing hematopoietic stem-cell transplantation or chemotherapy for hematologic cancers or solid tumors and it was expected that they would have platelet counts of 10,000 per cubic millimeter or lower for 5 days or more. Additional criteria were a weight of 10 to $135 \mathrm{~kg}$, prothrombin and partialthromboplastin times 1.3 times the upper limit of the normal range or less, a fibrinogen level of $100 \mathrm{mg}$ per deciliter or more, and no previous platelet transfusions for thrombocytopenia during the current period of hospitalization.

Exclusion criteria were as follows: bleeding of grade 2 or higher, according to the World Health Organization (WHO) bleeding scale ${ }^{9}$ (ranging from grades 1 through 4 , with higher grades indicating worse bleeding; see below and the Supplementary Appendix, available with the full text of this article at NEJM.org) before or at the time of assessment; performance of bedside platelet leukoreduction; platelet refractoriness within the past 30 days, according to local criteria; a panel-reactive HLA antibody level of $20 \%$ or more; acute promyelocytic leukemia, idiopathic or thrombotic thrombocytopenic purpura, or the hemolytic-uremic syndrome; planned prophylactic transfusion of platelets at platelet counts of more than 10,000 per cubic 
millimeter ${ }^{10}$; major surgery within the previous 2 weeks; use of drugs intended to affect platelet number or function; pregnancy; and previous enrollment in this platelet-dose trial (PLADO trial).

\section{STRATIFICATION AND RANDOMIZATION}

Body-surface area was calculated from height and weight. ${ }^{11}$ Patients were randomly assigned in a 1:1:1 ratio, by means of computer-generated permuted blocks, to receive platelet transfusions of one of three doses $-1.1 \times 10^{11}, 2.2 \times 10^{11}$, and $4.4 \times 10^{11}$ platelets per square meter per transfusion (low-dose group, medium-dose group, and high-dose group, respectively) - according to four treatment strata ${ }^{12}$ : allogeneic hematopoietic stem-cell transplantation, autologous or syngeneic hematopoietic stem-cell transplantation, chemotherapy for hematologic cancer, or chemotherapy for solid tumor. Treatment-group assignments were balanced within trial sites with the use of dynamic balancing. ${ }^{12}$ For typical adults, the medium dose is similar to a standard adult dose with regard to the number of platelets.

\section{TRANSFUSIONS}

The blood-transfusion service was given each patient's assigned dose and the allowable range, $\pm 25 \%$ of the assigned dose. Site staff were not told the patient's assigned dose, but differences in transfusion volume prevented complete blinding. Selection of platelets for transfusion to achieve the correct "attempted dose" was specified as follows. For apheresis platelets, the platelet count at the time of collection was used to select a partial unit, a single unit, or multiple units. For pooled platelet concentrates, the mean platelet count per concentrate, based on quality-control data, was used to determine the number of platelet concentrates to combine. For each transfusion, an "at-issue" platelet count was obtained to determine the actual dose transfused. HLA-selected platelets were transfused completely to avoid product wastage, regardless of the patient's assigned dose.

Platelets were transfused prophylactically if the morning count was 10,000 per cubic millimeter or lower (the "trigger" threshold). The patient's physician could change the transfusion trigger threshold or dose if required by clinical indications, with a return to study guidelines as soon as possible. Local practice determined the indications for red-cell transfusion. Platelets and red cells were leukoreduced by means of filtration.

\section{CLINICAL ASSESSMENTS}

Research staff performed daily assessments of bleeding using physical examinations, interviews with patients, and chart reviews for bleeding events. They also collected data on all bleeding described in the WHO criteria, ${ }^{9}$ except they did not perform urine dipstick or stool guaiac tests. The bleeding data were used to calculate each patient's daily bleeding grade. Daily platelet counts, hematocrit values, and hemoglobin levels were also measured.

The primary end point was bleeding of grade 2 or higher. Grade 2 bleeding was defined as oropharyngeal bleeding or epistaxis for more than 30 minutes during a 24-hour period, purpura of more than $1 \mathrm{in} .(2.54 \mathrm{~cm})$ in diameter, deep hematoma, joint bleeding, melena, hematochezia, hematemesis, gross hematuria, abnormal vaginal bleeding consisting of more than spotting, hemoptysis, blood in bronchopulmonary lavage specimens, visible blood in body-cavity fluid without symptoms, retinal bleeding without visual impairment, spinal-fluid specimens containing microscopic amounts of blood, or bleeding for more than 1 hour at invasive sites. ${ }^{9}$ (See the Supplementary Appendix for definitions of bleeding grades 0 through 4.) If an investigator indicated possible death from hemorrhage or if a deceased patient had had grade 3 or 4 bleeding, three nonstudy physicians adjudicated whether bleeding was the cause of death. 


\section{ADVERSE EVENTS}

Information was collected on all serious adverse events and on events commonly associated with transfusion that occurred during transfusion or within 4 hours afterward.

\section{STUDY COMPLETION}

The study was considered to be completed at 30 days after the first platelet transfusion, after a 10-day period without a platelet transfusion, at hospital discharge, at death, or at withdrawal from the study — whichever occurred first.

\section{STATISTICAL ANALYSIS}

The primary end point was bleeding of grade 2 or higher; secondary end points were the highest grade of bleeding, total number of platelets transfused, and number of platelet transfusions. Other analyses were exploratory. To account for the three pairwise comparisons among the three treatment groups, two-sided $\mathrm{P}$ values of less than 0.017 were considered to indicate statistical significance. No other adjustment was made for analyzing multiple outcomes. The study was designed to have a statistical power of $85 \%$ to detect an absolute difference of $12.5 \%$ in the incidence of the primary end point for any pair among the three treatment groups, which required 450 patients per group.

Unless otherwise specified, all analyses were restricted to data for patients who received at least one platelet transfusion. Results were analyzed according to the patient's treatment assignment, even if platelet transfusions were not received at the assigned dose or were not administered in accordance with the prophylactic transfusion trigger threshold of 10,000 platelets per cubic millimeter.

Dichotomous end points were compared among the groups with the use of Fisher's exact test. The highest grade of bleeding was compared using an exact version of the Kruskall-Wallis test. The time to next transfusion and time to bleeding were compared among the groups by means of the log-rank test. ${ }^{13}$ Dose adherence for each transfusion, trigger-threshold adherence for each study day, pretransfusion and post-transfusion platelet-counts, platelet increments, and corrected count increments were compared among the groups with the use of generalized linear models to account for possible correlations between results within each patient. ${ }^{14,15}$ (The platelet increment is defined as the post-transfusion platelet count minus the pretransfusion count; the corrected count increment has a numerator of the platelet increment [in cubic millimeters] multiplied by the body-surface area [in square meters] and a denominator of the total number of platelets transfused divided by $10^{11}$.) Other continuous variables were analyzed with the use of the Wilcoxon rank-sum test. The post hoc subgroup analysis of data regarding the primary end point, according to randomization stratum, used a logistic-regression model with an interaction term.

\section{RESULTS \\ STUDY POPULATION}

Between 2004 and 2007, a total of 1351 patients were enrolled at 26 sites. Seventy-nine patients did not receive a platelet transfusion; however, including these patients in the analyses had a negligible effect on the results. The baseline characteristics of the study patients were well balanced among the three treatment groups (Table 1).

\section{ADHERENCE WITH PLATELET DOSE AND TRANSFUSION TRIGGER THRESHOLD}

Dose adherence was not required for 210 HLA-selected units of platelets and could not be reliably determined for an additional 331 volume-reduced units from which plasma was 
removed by centrifugation after collection. Among the 5466 prophylactic platelet transfusions with neither characteristic, the attempted dose was known for 5384 transfusions in 1162 patients - and there was dose adherence for $86 \%$ of these low-dose transfusions, $98 \%$ of these medium-dose transfusions, and $93 \%$ of these high-dose transfusions ( $\mathrm{P}<0.001$ for low dose vs. medium dose and medium dose vs. high dose) (Table 2). Among the 1162 patients, all known attempted doses were in adherence for $79 \%, 92 \%$, and $86 \%$ of patients in the low-dose, medium-dose, and high-dose groups, respectively ( $\mathrm{P}<0.001$ for low dose vs. medium dose, and $\mathrm{P}=0.004$ for medium dose vs. high dose).

Platelet doses based on at-issue platelet counts were within the patient's assigned range for $71 \%, 80 \%$, and $70 \%$ of transfusions in the low-dose group, medium-dose group, and high-dose group, respectively ( $\mathrm{P}=0.007$ for low dose vs. medium dose, and $\mathrm{P}<0.001$ for medium dose vs. high dose). This result confirms that the platelet-selection procedure usually resulted in transfusions of at-issue doses in the assigned range. All known at-issue doses were within the assigned range for $51 \%, 63 \%$, and $55 \%$ of patients in the low-dose, medium-dose, and highdose groups, respectively $(\mathrm{P}<0.001$ for low dose vs. medium dose).

Physicians ordered changes in the platelet dose for clinical reasons for $17 \%$ of patients in the low-dose group, $9 \%$ in the medium-dose group, and 7\% in the high-dose group $(\mathrm{P}=0.003$ for low dose vs. medium dose and $\mathrm{P}<0.001$ for low dose vs. high dose). Overall, only $3 \%$ of patients had a dose change before the onset of bleeding of grade 2 or higher.

The trigger threshold of 10,000 platelets per cubic millimeter was adhered to on $90 \%, 92 \%$, and $94 \%$ of patient-days in the low-dose group, medium-dose group, and high-dose group, respectively ( $\mathrm{P}<0.001$ for low dose vs. high dose). The trigger was adhered to on all study days for $53 \%, 62 \%$, and $61 \%$ of patients, respectively ( $\mathrm{P}=0.01$ for low dose vs. medium dose). Physicians ordered changes in the trigger threshold for clinical reasons for $32 \%, 26 \%$, and $25 \%$ of patients, respectively, but a total of only $7 \%$ of patients had a change in the trigger threshold before the onset of bleeding of grade 2 or higher.

\section{PRIMARY AND SECONDARY END POINTS}

In the 1351 patients who underwent randomization, the percentage with at least one episode of bleeding of grade 2 or higher was $68 \%$ in the low-dose group, $67 \%$ in the medium-dose group, and $69 \%$ in the high-dose group, with no significant differences among the groups $(\mathrm{P}$ $=0.83$ for low dose vs. medium dose and for low dose vs. high dose $\mathrm{P}=0.66$ for medium dose vs. high dose). Among the 1272 patients who received at least one platelet transfusion, the percentage of patients in each group with at least one episode of bleeding of grade 2 or higher was not significantly different $(71 \%, 69 \%$ and $70 \%$, respectively) (Table 3$)$.

Overall, the highest bleeding grade observed was no bleeding or bleeding of grade 1 in $31 \%$ of patients, grade 2 in $59 \%$, grade 3 in $8 \%$, and grade 4 in $2 \%$. In each group, the median number of days of bleeding of grade 2 or higher was 1 . The median time from randomization to onset of bleeding ranged from 7 to 8 days. The median number of red-cell units transfused per patient was 4 . A total of $93 \%$ of patients received at least one red-cell transfusion. There were no significant differences among the three groups for any of these end points.

Bleeding of grade 2 or higher occurred in $79 \%$ of recipients of allogeneic hematopoietic stemcell transplants, $73 \%$ of patients who had hematologic cancers and were undergoing chemotherapy, and 57\% of patients undergoing autologous or syngeneic hematopoietic stemcell transplantation $(\mathrm{P}<0.001$ for the comparison of the latter group with each of the first two groups). However, within each of these treatment categories, the platelet dose had no significant effect on bleeding. 
The 1272 patients who received at least one transfusion were observed for a total of 24,309 days. Figure 1 shows the percentage of days on which bleeding of grade 2 or higher occurred, according to the patient's morning platelet-count category. Bleeding of grade 2 or higher occurred on $25 \%$ of days with morning platelet counts of 5000 per cubic millimeter or lower, $17 \%$ of days with morning platelet counts from 6000 to 80,000 per cubic millimeter, $13 \%$ of days with morning platelet counts from 81,000 to 100,000 per cubic millimeter, and $8 \%$ of days with morning platelet counts over 100,000 per cubic millimeter $(\mathrm{P}<0.001$ for platelet counts of $\leq 5000$ per cubic millimeter vs. counts of 6000 to 80,$000 ; P=0.001$ for platelet counts of 81,000 to 100,000 per cubic millimeter vs. counts of 6000 to 80,000 ; and $\mathrm{P}<0.001$ for platelet counts of $>100,000$ per cubic millimeter vs. counts of 6000 to 80,000) (Fig. 1).

We could calculate the total number of platelets transfused per patient for 1000 patients. The median number of platelets transfused was $9.25 \times 10^{11}, 11.25 \times 10^{11}$, and $19.63 \times 10^{11}$ in the lowdose, medium-dose, and high-dose groups, respectively $(\mathrm{P}=0.002$ for low dose vs. medium dose, $\mathrm{P}<0.001$ for high dose vs. low dose and high dose vs. medium dose) (Table 3 ). The median number of platelet transfusions administered was five in the low-dose group as compared with three in the medium-dose group and three in the high-dose group $(\mathrm{P}<0.001$ for low dose vs. medium dose and low dose vs. high dose).

\section{RESPONSES TO PLATELET TRANSFUSIONS}

The low-dose, medium-dose, and high- dose groups differed significantly in the median post -transfusion platelet count $(22,000,34,000$, and 50,000 per cubic millimeter, respectively), the median increase in platelet count after transfusion $(10,000,19,000$, and 38,000 per cubic millimeter, respectively), and the median number of days until the next transfusion (1.1, 1.9, and 2.9, respectively) $(\mathrm{P}<0.001$ for all comparisons for all end points) (Table 2$)$. The median 4-hour corrected count increment was 10,000 in the low-dose group, 10,000 in the mediumdose group, and 11,000 in the high-dose group, with no significant differences among groups.

\section{ADVERSE EVENTS}

There were no significant differences among the three groups in the occurrence of any specific category of serious adverse events or in the percentage of patients who had one or more serious adverse events (Table 4). Wheezing during or shortly after transfusion was significantly more common in the high-dose group than in the medium-dose group. Nine patients in the low-dose group died, as did four patients in the medium-dose group and seven in the high-dose group; the number of deaths did not differ significantly among the three groups.

\section{STUDY COMPLETION}

Completion of the study occurred at the time of hospital discharge for most patients (71\%). Other causes of study completion were an absence of platelet transfusion for 10 days (in 14\% of patients), the elapsing of 30 days from first platelet transfusion (10\%), withdrawal from the study (4\%), and death (2\%). The three groups did not differ significantly with regard to reasons for study completion.

\section{DISCUSSION}

This study evaluated the effects of platelet dose on hemostasis and transfusion end points. For a typical adult, the medium dose was equivalent to the standard dose currently used in clinical practice. ${ }^{16}$ The low dose was half the medium dose, and the high dose was twice the medium dose. There were high rates of adherence for platelet doses and transfusion trigger thresholds. Physicians changed the dose to a nonstudy dose for patients in the low-dose group more often than for those in the medium-dose and high-dose groups, but the changes were made primarily after the onset of bleeding of grade 2 or higher. 
The percentages of patients who received at least one platelet transfusion and had bleeding of grade 2 or higher $-71 \%, 69 \%$, and $70 \%$ in the low-, medium-, and high-dose groups, respectively - were not significantly different. The only death from hemorrhagic causes occurred in the high-dose group (from pulmonary hemorrhage). The highest grade of bleeding, the number of days of bleeding of grade 2 or higher, the number of days before the occurrence of bleeding of grade 2 or higher, and the occurrence and number of red-cell transfusions also did not differ significantly among the three groups. These findings confirm that the dose per prophylactic platelet transfusion, within the range of doses studied, did not significantly affect bleeding.

The percentage of patients with bleeding of grade 2 or higher was significantly less among those undergoing autologous or syngeneic hematopoietic stem-cell transplantation (57\%) than among those with hematologic cancers who were undergoing chemotherapy (73\%) or those undergoing allogeneic hematopoietic stem-cell transplantation (79\%), as has been described previously. ${ }^{17}$ However, platelet dose had no significant effect on bleeding in any of these treatment categories.

Patients had a 25\% risk of having bleeding of grade 2 or higher on days on which the morning platelet count was 5000 per cubic millimeter or lower, as compared with a $17 \%$ risk on days with counts from 6000 to 80,000 per cubic millimeter. Previous reports suggest that endothelial integrity can be maintained with platelet counts of 5000 per cubic millimeter or higher. ${ }^{18,19}$ Further reductions in the risk of bleeding at platelet counts of 80,000 per cubic millimeter or higher are postulated to be due to an improved clinical status.

The rates of bleeding seen in our trial are higher than those in several other platelet-transfusion trials. ${ }^{20-22}$ The reported incidence of bleeding depends on factors that often differ among studies, such as assessment method, frequency of assessment, criteria used for bleeding grade, and population of patients. ${ }^{20}$ In our study, consistent data collection was achieved with the use of daily hemostatic assessments performed by research staff who were unaware of the treatment assignments and who followed detailed instructions. Bleeding grades were ascertained on the basis of objective criteria, thus reducing bias.

To our knowledge, only two previously reported randomized trials, with limited enrollment, have evaluated the use of low-dose platelet transfusions as compared with standard-dose transfusions. ${ }^{5,6}$ Neither trial showed any significant differences regarding bleeding of any WHO grades - findings similar to those in our study.

The Strategies for Transfusion of Platelets (SToP) study (NCT00420914) ${ }^{6}$ was halted because of grade 4 bleeding in three patients in the low-dose group $\left(1.5 \times 10^{11}\right.$ to $3.0 \times 10^{11}$ platelets per transfusion), as compared with none in the standard-dose group $\left(3.0 \times 10^{11}\right.$ to $6.0 \times 10^{11}$ platelets per transfusion). A stopping rule of a 5\% absolute difference between the two groups in the incidence of grade 4 bleeding was reached after only 58 and 61 patients were enrolled in the low-dose group and the standard-dose group, respectively. We did not find a significant difference in the incidence of grade 4 bleeding in our similar, but much larger, cohort. An important difference between our study and the StoP study was that we adjusted the dose of platelets for body-surface area, whereas in the StoP study, the same dose range was used for all patients in each of the two groups.

In our trial, the total number of platelets transfused $-9.25 \times 10^{11}$ for the low dose, $11.25 \times 10^{11}$ for the medium dose, and $19.63 \times 10^{11}$ for the high dose - was significantly different among the three groups. However, the median number of transfusions per patient was significantly greater in the low-dose group (five transfusions) than in the medium-or high-dose group (three transfusions in each group) ( $\mathrm{P}<0.001$ for both comparisons). In the SToP study, 
6 the low-dose group also required significantly more transfusions than the standard-dose group.

As expected, the median platelet increment after transfusion in the low-dose group $(10,000$ per cubic millimeter) was approximately half the median increment in the medium-dose group $(19,000$ per cubic millimeter), and the median increment in the high-dose group $(38,000$ per cubic millimeter) was approximately twice the increment in the medium-dose group. The number of days until the next transfusion also differed significantly among the three groups: 1.1 days in the low-dose group, 1.9 in the medium-dose group, and 2.9 in the high-dose group. These data, data from other, smaller studies, and mathematical models all suggest that larger doses give higher increments and prolonged intervals until the next transfusion. ${ }^{3,23-27}$ However, the corrected count increment did not differ significantly among the three groups (i.e., differences in the increment were explained by differences in the number of platelets transfused).

In conclusion, when prophylactic transfusions are given after a trigger threshold of 10,000 platelets per cubic millimeter or lower is reached, the platelet dose has no significant effect on the incidence of bleeding in patients with hypoproliferative thrombocytopenia, probably because few platelets are needed to maintain hemostasis. ${ }^{18,19}$ A strategy of low-dose transfusion significantly reduces the quantity of platelets transfused, which could preserve these scarce blood components but could also increase the number of platelet transfusions.

\title{
Supplementary Material
}

Refer to Web version on PubMed Central for supplementary material.

\section{Acknowledgments}

\begin{abstract}
Supported by grants from the National Heart, Lung, and Blood Institute of the National Institutes of Health to the Data Coordinating Center at New England Research Institutes (HL072268), Case Western Reserve University (HL072033), Children's Hospital Boston (HL072291), Cornell University (HL072196), Duke University (HL072289), Emory University (HL072248), Johns Hopkins University (HL072191), Massachusetts General Hospital (HL072299), Puget Sound Blood Center (HL072305), Tulane University (HL072274), University of Iowa (HL072028), University of Maryland (HL072359), University of Minnesota (HL072072), University of North Carolina (HL072355), University of Oklahoma (HL072283), University of Pennsylvania (HL072346), University of Pittsburgh (HL072331), and the Blood Center of Wisconsin (HL072290).
\end{abstract}

Dr. Slichter reports receiving grant support from U.S. Army Medical Research Acquisition Activity (Department of Defense), Navigant Biotechnologies, and Pall Medical; Dr. Assmann, receiving grant support from Z-Medica; Dr. Triulzi, receiving consulting fees from Fenwal Laboratories and Cerus and lecture fees from Pall; Dr. Strauss, receiving consulting fees from CaridianBCT; Dr. Ness, receiving consulting fees from Fenwal Laboratories and CaridianBCT; Dr. Brecher, receiving consulting fees from Fenwal Laboratories; Dr. Josephson, receiving lecture fees from Mediware; Dr. George, receiving consulting fees and grant support from Amgen; and Dr. Manno, receiving consulting fees and grant support from Baxter Healthcare, consulting fees from Bayer Healthcare, and lecture fees from EMD Healthcare Communications Scientific Communication Group. No other potential conflicts of interest relevant to this article were reported.

We thank all the patients who volunteered to take part in this study, all the study investigators and committee members, and Ginny Knight at the Puget Sound Blood Center for administrative assistance.

\section{APPENDIX}

The following investigators and staff participated in the study: Case Western Reserve

University, Cleveland: K.R. McCrae (principal investigator), V. Exum, D. Hendrix; BloodCenter of Wisconsin, Milwaukee, and University of Wisconsin-Madison, Madison: J.G. McFarland (principal investigator), R.D. Woodson, N. Ruys, J. Werndli, K. Koenig, N. Turman, J. Stublaski; Froedert Memorial Lutheran Hospital, Milwaukee: M. 
Lankiewicz, D. Miller-Metcalfe, S. Graminske, R. Dora, S. Heldke; St. Luke's Medical Center, Milwaukee: A.B. Divgi, J. Boos, S. Beekman; Children's Hospital of Wisconsin, Milwaukee: R. Punzalan; Children's Hospital Boston, Boston: E.J. Neufeld (principal investigator), S.R. Sloan, T. Kang, J. Hedstrom, K. Harney; Beth Israel Deaconess Medical Center, Boston: L. Uhl, B. Malynn; Brigham and Women's Hospital, Boston: R.M. Kaufman, M. Kelly, B. Rowe, K. Wallace, S. Slate; Weill College of Cornell University, New York: J. Bussel (principal investigator), D.L. Skerrett, E. Feldman, C. Nguyen, J. Cruz, M. Wissert; Duke University, Durham, NC: T.L. Ortel (principal investigator), S. Adams, T. Anderson, C. Higgins, C. Mette, E. Petzold, C. Pinaroc, C. Thornburg, M.J. Telen; Emory University, Atlanta: C.D. Hillyer (principal investigator), C.D. Josephson, M.-I. Castillejo, J. Newman, J. Ieong, J. Johnson; Johns Hopkins University, Baltimore: P.M. Ness (principal investigator), R. Case, A. Fuller, A.K. Fuller; Puget Sound Blood Center and University of Washington Medical Center, Seattle: S.J. Slichter (principal investigator), T.B. Gernsheimer, A. Deyle, J. Corson, A. Hirata; Virginia Mason Medical Center, Seattle: D. Aboulafia; Children's Hospital Medical Center, Seattle: D. Matthews; Tulane University Hospital and Clinics, New Orleans: C. Leissinger (principal investigator), R. Kruse-Jarres, H. Safah, C. Schmidt; University of Iowa, Iowa City: R.G. Strauss (principal investigator), B. Link, T. Raife, J. Swift, D. Schrock; University of Maryland, Baltimore: J.R. Hess (principal investigator), M. Mitrou, J. Spanfelner; Fairview-University Medical Center, Minneapolis: J. McCullough (principal investigator), T. Carr, T.Chlebeck, S. Malcolm, S. Pulkrabek; University of North Carolina, Chapel Hill: M.E. Brecher (principal investigator), A. Tsui, M. Miller, S. Hay; University of Oklahoma, Oklahoma City: J.N. George (principal investigator), D. Terrell, X. Li; University of Texas Southwestern Medical Center, Dallas: G. Buchanan (principal investigator), V.M. Aquino, J. Cox, T. Hoffman, G. Paranjape; University of Pennsylvania, Philadelphia: B.A. Konkle (principal investigator), E.A. Stadtmauer, I. Tarng, M. Kelty, M. Einarson, K. Hinkle; Children's Hospital of Philadelphia, Philadelphia: C.S. Manno (principal investigator), A. Parker, A. Wade, J. Nathanson, M. Braun; University of Pittsburgh, Pittsburgh: D.J. Triulzi (principal investigator), P. D'Andrea, L. Blanker; Dartmouth-Hitchcock Medical Center, Lebanon, NH: J. AuBuchon (medical monitor); New England Research Institutes (Data Coordinating Center), Watertown, MA: S.F. Assmann (principal investigator), D. Brambilla, A.S. Corbett, E. Devlin, J. Erickson, E. Gerstenberger, S. Granger, K. Hayes, J. Miller, J. Scott; National Heart, Lung, and Blood Institute, Bethesda, MD: G. Nemo (project officer), S. Glynn, L. Harvath, E. Leifer, T. Mondoro, E. Wagner; Data and Safety Monitoring Board: S. Geyer, T. Lane, J. Lusher, B. McLeod, A. Reitsma, P. Roberson, A. Shapiro, C. Whitsett.

\section{REFERENCES}

1. Tinmouth AT, Freedman J. Prophylactic platelet transfusions: which dose is the best dose? A review of the literature. Transfus Med Rev 2003;17:181-193. [PubMed: 12881779]

2. Heddle NM. Controversy concerning platelet dose. ISBT Sci Ser 2007;2:220-225.

3. Stanworth SJ, Hyde C, Brunskill S, Murphy MF. Platelet transfusion prophylaxis for patients with haematological malignancies: where to now? Br J Haematol 2005;131:588-595. [PubMed: 16351634]

4. Norol F, Bieling P, Roudot-Thoraval F, et al. Platelet transfusion: a dose-response study. Blood 1998;92:1448-1453. [PubMed: 9694735]

5. Tinmouth A, Tannock IF, Crump M, et al. Low-dose prophylactic platelet transfusions in recipients of an autologous peripheral blood progenitor cell transplant and patients with acute leukemia: a randomized controlled trial with a sequential Bayesian design. Transfusion 2004;44:1711-1719. [PubMed: 15584985]

6. Heddle NM, Cook RJ, Tinmouth A, et al. A randomized controlled trial comparing standard-and lowdose strategies for transfusion of platelets (SToP) to patients with thrombocytopenia. Blood 2009;113:1564-1573. [PubMed: 19109560] 
7. Slichter SJ. Background, rationale, and design of a clinical trial to assess the effects of platelet dose on bleeding risk in thrombocytopenic patients. J Clin Apher 2006;21:78-84. [PubMed: 16619231]

8. SAS/STAT software, version 9.2. Cary, NC: SAS Institute; 2000-2008.

9. Miller AB, Hoogstraten B, Staquet M, Winkler A. Reporting results of cancer treatment. Cancer 1981;47:207-214. [PubMed: 7459811]

10. Rebulla P, Finazzi G, Marangoni F, et al. The threshold for prophylactic platelet transfusions in adults with acute myeloid leukemia. N Engl J Med 1997;337:1870-1875. [PubMed: 9407153]

11. DuBois D, DuBois EF. A formula to estimate the approximate surface area if height and weight be known. Arch Intern Med 1916;17:863-871.

12. Zelen M. The randomization and stratification of patients to clinical trials. J Chronic Dis 1974;27:365375. [PubMed: 4612056]

13. Cox DR, Oakes D. Analysis of survival data. London: Chapman \& Hall. 1984

14. Hardin, JW.; Hilbe, JM. Generalized estimating equations. Boca Raton, FL: Chapman \& Hall/CRC; 2003.

15. Liang K-Y, Zeger SL. Longitudinal data analysis using generalized linear models. Biometrika 1986;73:13-22.

16. Whitaker, BI.; Green, J.; King, MR., et al. The 2007 National Blood Collection and Utilization Survey report. (Accessed January 25, 2010, at. http://www.hhs.gov/ophs/bloodsafety/2007nbcus_survey.pdf.)

17. Nevo S, Swan V, Enger C, et al. Acute bleeding after bone marrow transplantation (BMT ) incidence and effect on survival: a quantitative analysis in 1,402 patients. Blood 1998;91:1469-1477. [PubMed: 9454780]

18. Slichter SJ. Relationship between platelet count and bleeding risk in thrombocytopenic patients. Transfus Med Rev 2004;18:153-167. [PubMed: 15248165]

19. Heddle NM, Cook RJ, Sigouin C, Slichter SJ, Murphy M, Rebulla P. A descriptive analysis of international transfusion practice and bleeding outcomes in patients with acute leukemia. Transfusion 2006;46:903-911. [PubMed: 16734806]

20. Heddle NM, Cook RJ, Webert KE, Sigouin C, Rebulla P. Methodologic issues in the use of bleeding as an outcome in transfusion medicine studies. Transfusion 2003;43:742-752. [PubMed: 12757525]

21. Wandt H, Frank M, Schaefer-Eckart K, Wilhelm M. Routine prophylactic platelet transfusions are not necessary in patients with acute myeloid leukemia — a therapeutic transfusion strategy is safe and cost effective. Blood 2005;106:129a. abstract.

22. McCullough J, Vesole DH, Benjamin RJ, et al. Therapeutic efficacy and safety of platelets treated with a photochemical process for pathogen inactivation: the SPRINT trial. Blood 2004;104:15341541. [PubMed: 15138160]

23. Hersh JK, Hom EG, Brecher ME. Mathematical modeling of platelet survival with implications for optimal transfusion practice in the chronically platelet transfusion-dependent patient. Transfusion 1998;38:637-644. [PubMed: 9683101]

24. Brecher ME, Hom EG, Hersh JK. Optimal platelet dosing. Transfusion 1999;39:431-434. [PubMed: 10220274]

25. Klumpp TR, Herman JH, Gaughan JP, et al. Clinical consequences of alterations in platelet transfusion dose: a prospective, randomized, double-blind trial. Transfusion 1999;39:674-681. [PubMed: 10413273]

26. Sensebé L, Giraudeau B, Bardiaux L, et al. The efficiency of transfusing high doses of platelets in hematologic patients with thrombocytopenia: results of a prospective, randomized, open, blinded end point (PROBE) study. Blood 2005;105:862-864. [PubMed: 15367427]

27. Goodnough LT, Kuter DJ, McCullough J, et al. Prophylactic platelet transfusions from healthy apheresis platelet donors undergoing treatment with thrombopoietin. Blood 2001;98:1346-1351. [PubMed: 11520781] 


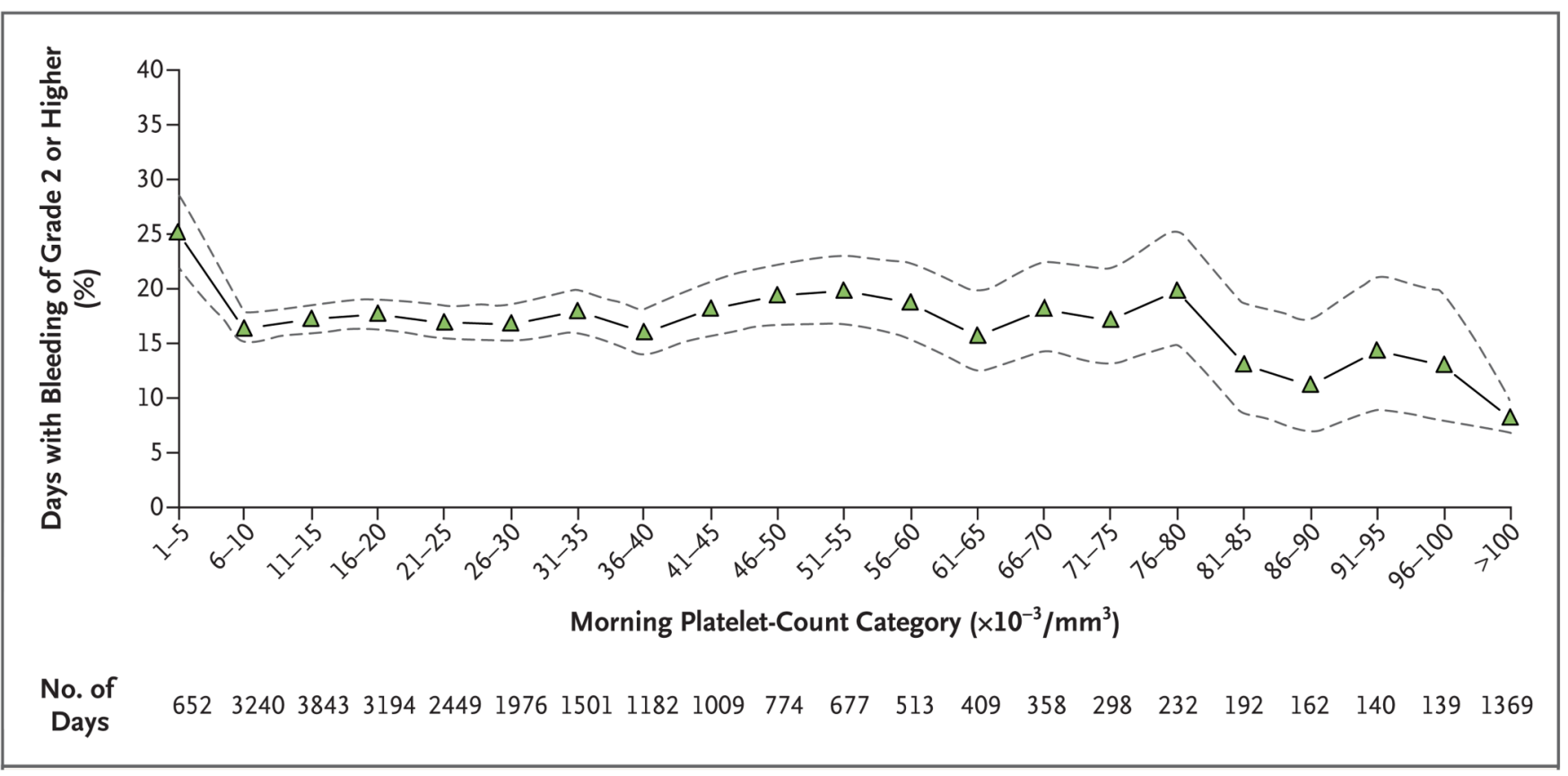

Figure 1. Days with Bleeding of Grade 2 or Higher in All three Treatment Groups, According to Morning Platelet-Count Categories

The percentage of days on which patients had bleeding of grade 2 or higher is shown, along with the associated 95\% confidence intervals (dashed lines), according to the morning plateletcount category. Data are based on the 24,309 days during the study period on which patients had both a morning platelet count and information on bleeding of grade 2 or higher. Each patient-day was treated as a separate unit of analysis. Analyses were adjusted to take into account that for each patient, the results on various days may be correlated. The interaction between treatment group and morning platelet-count category was not significant, indicating that the effect of the morning platelet-count category did not differ significantly among the three treatment groups; therefore, the data from all three groups are combined. 


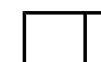

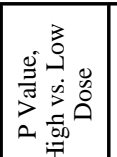
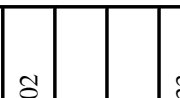

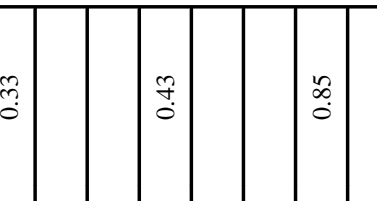

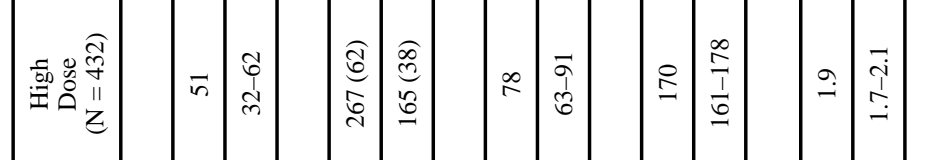

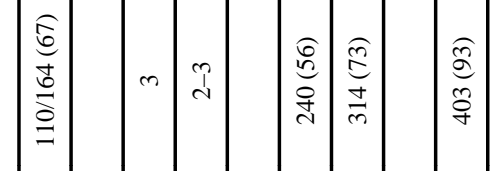

()

离

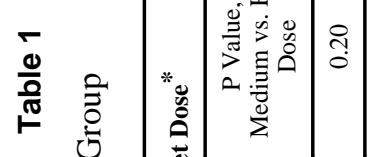

$\stackrel{\infty}{\infty}$

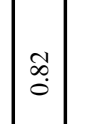

ลุ่

告

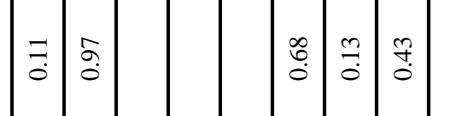

竧密

in $\mid \begin{aligned} & \infty \\ & \infty \\ & m \\ & m\end{aligned}$

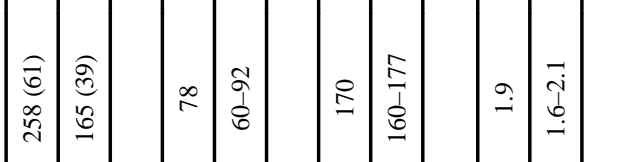

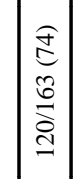

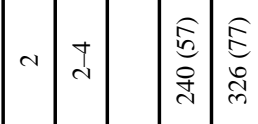

商

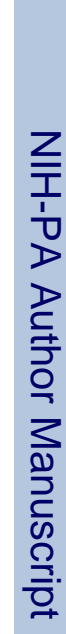

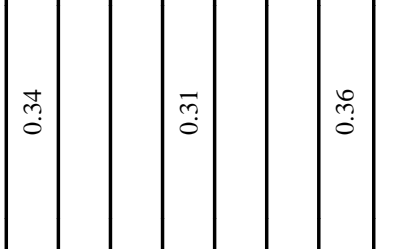
守 寺 $+\quad$ :

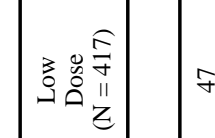

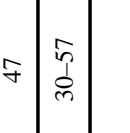

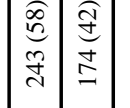

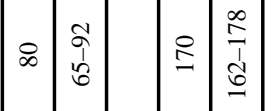

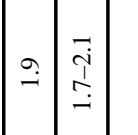
¿ें

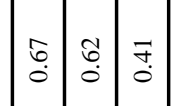

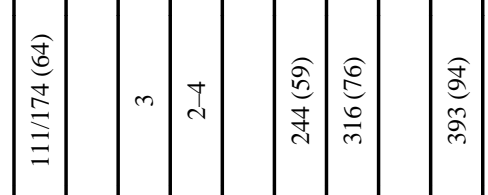

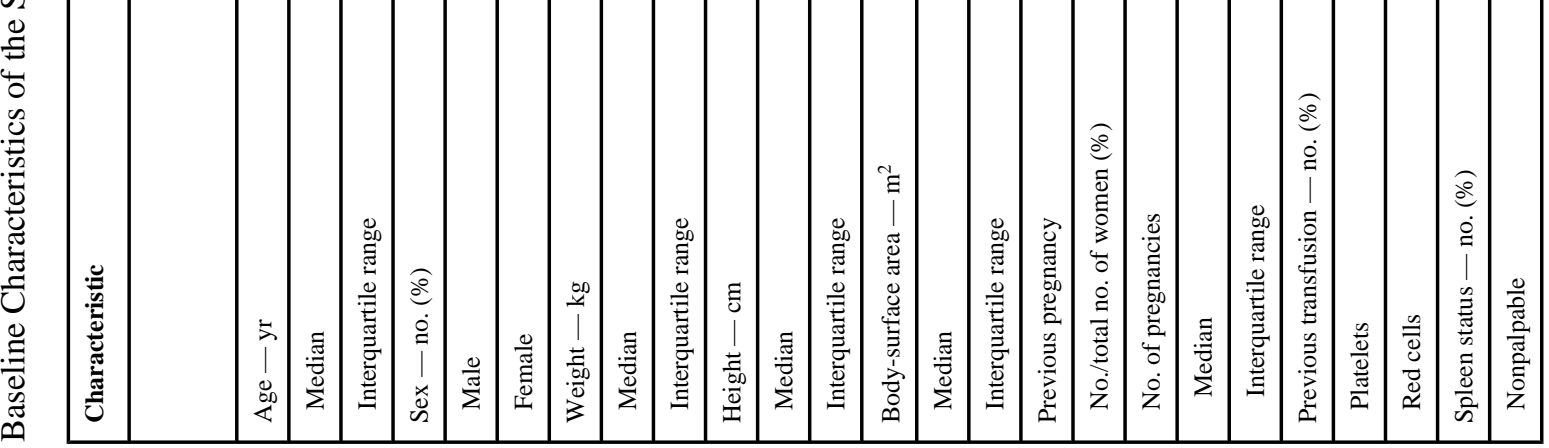




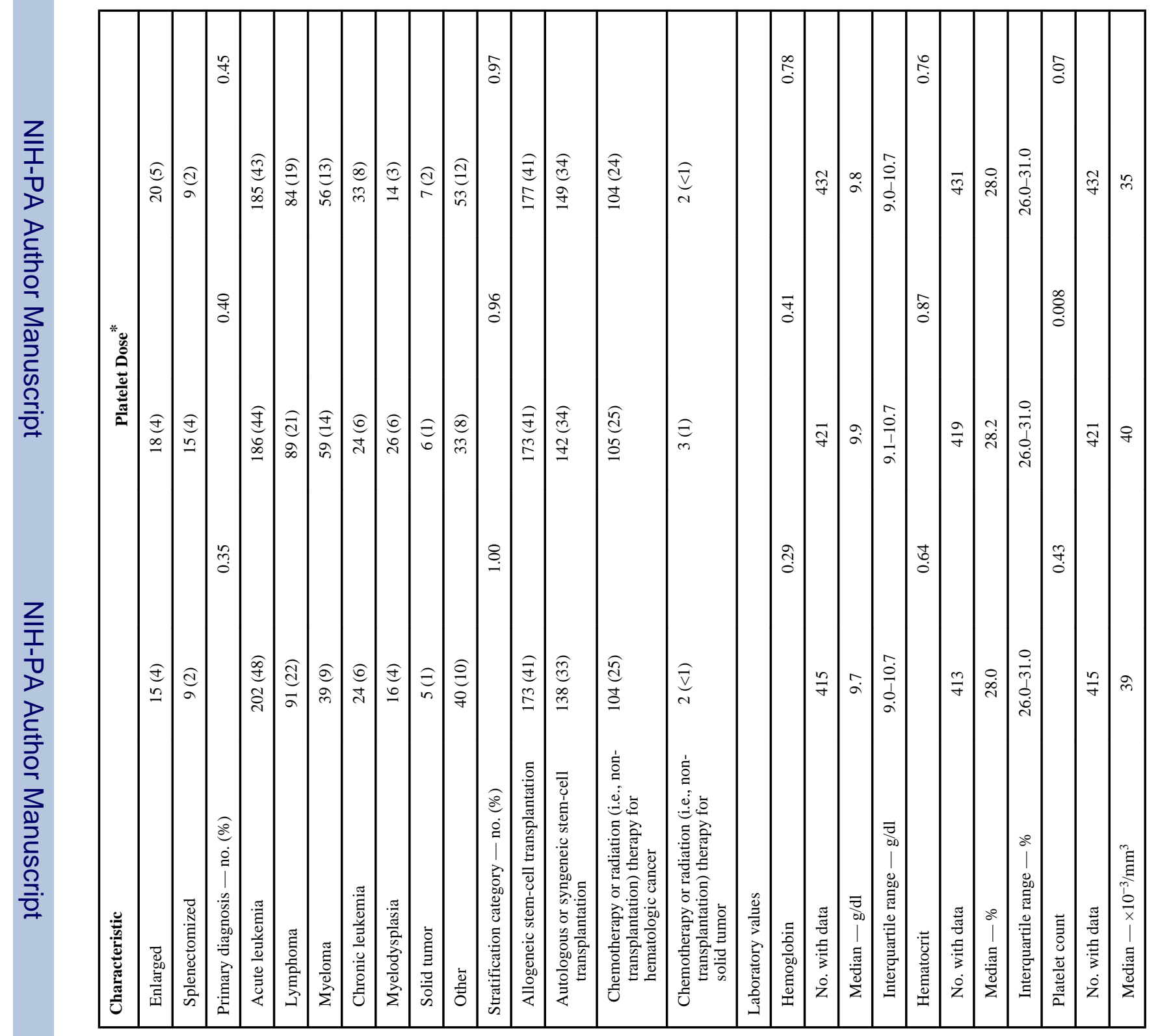

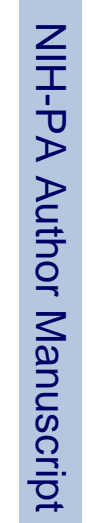




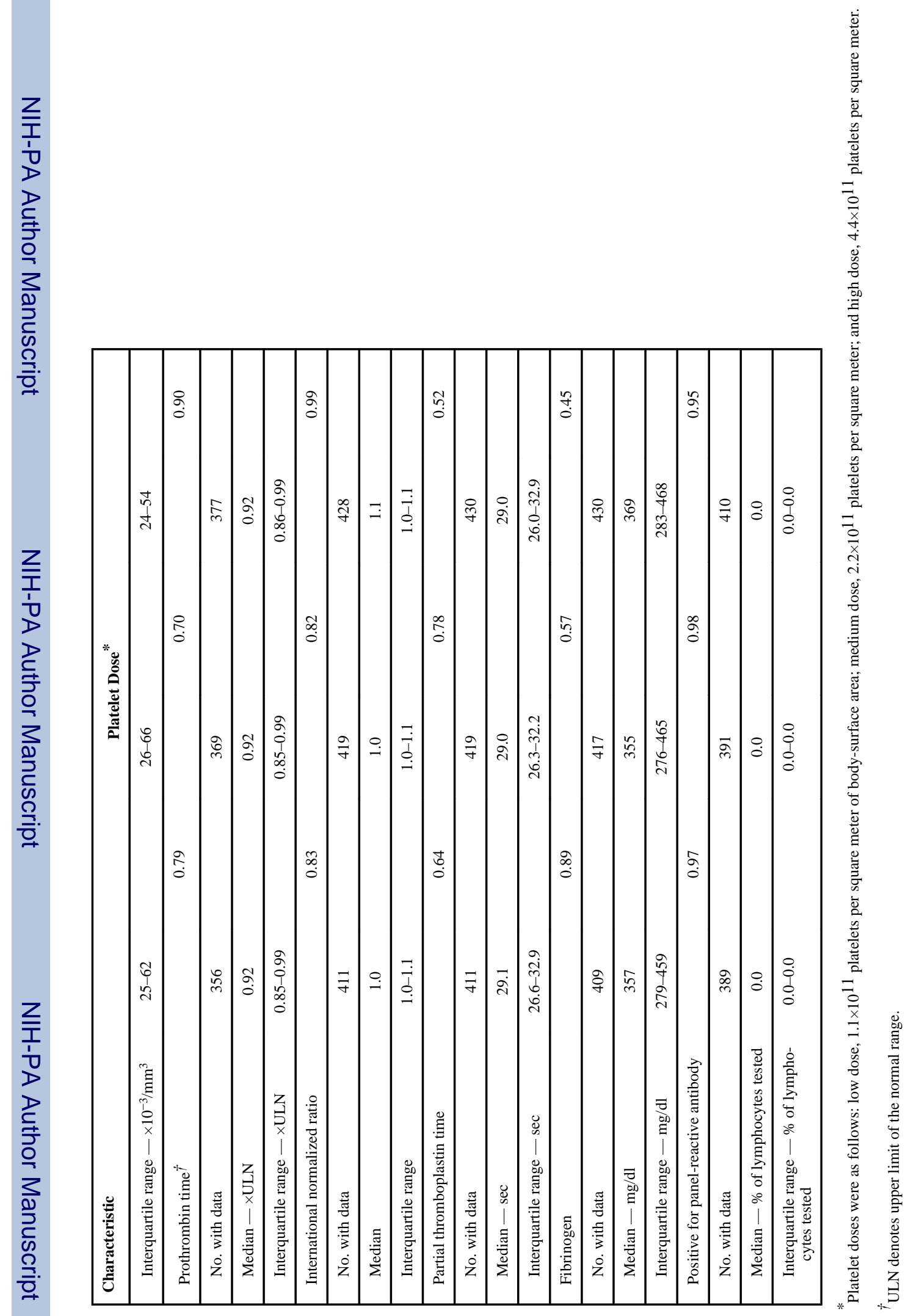




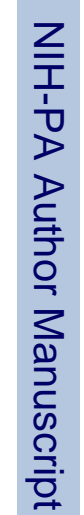

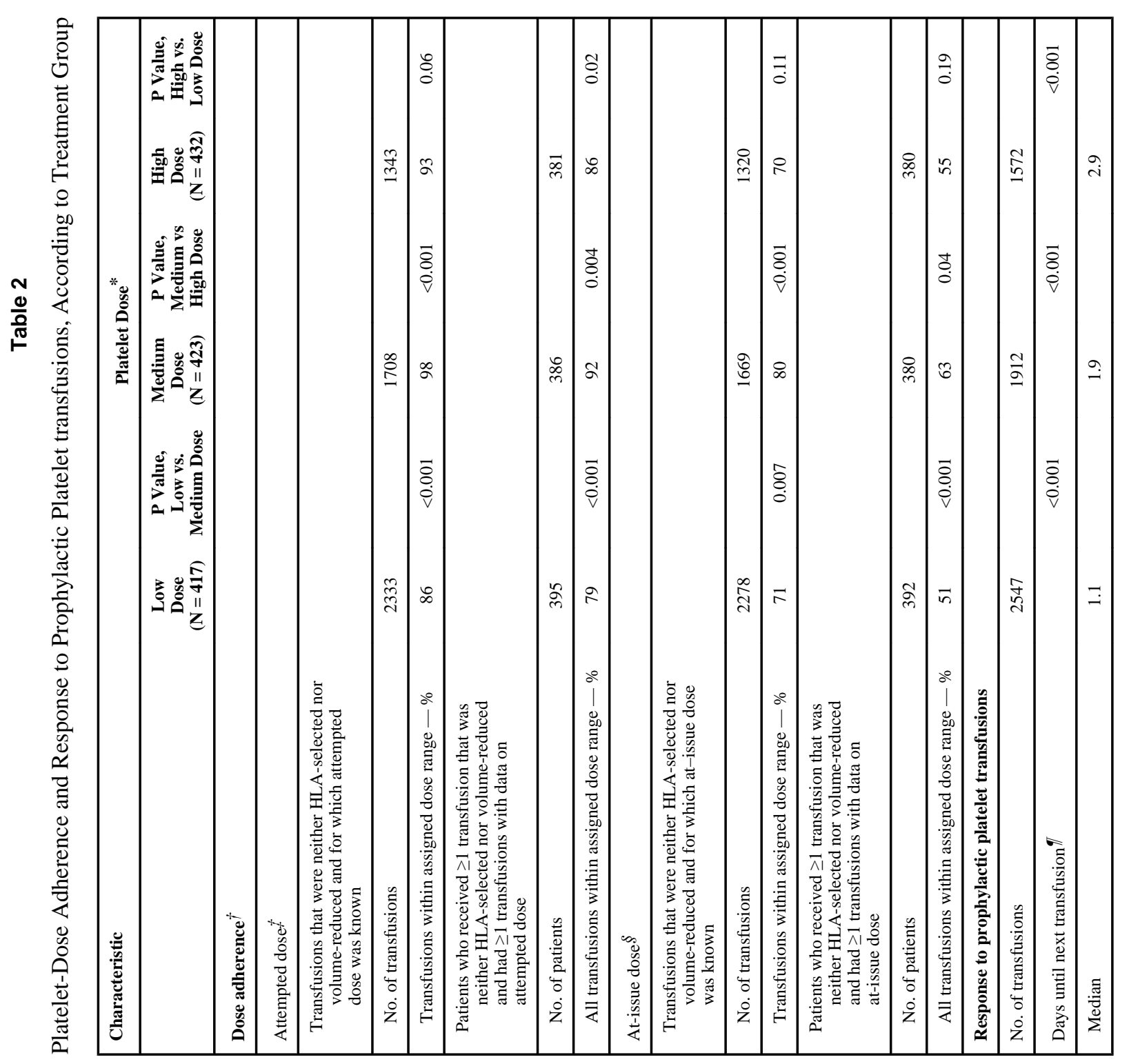

N Engl J Med. Author manuscript; available in PMC 2010 October 7. 


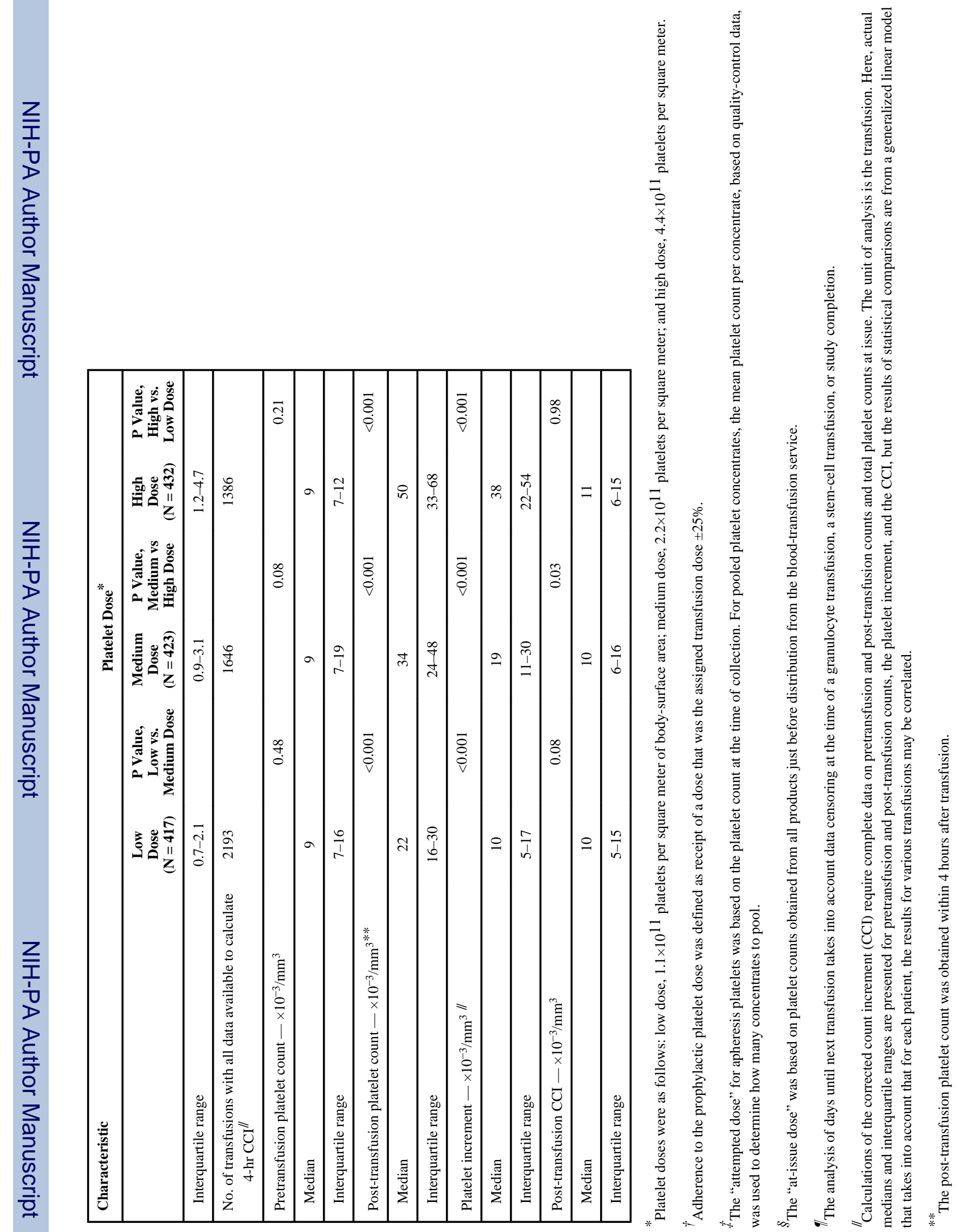




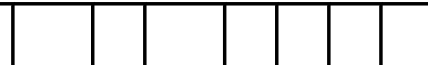

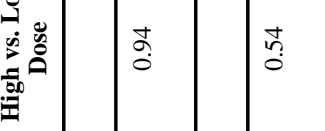

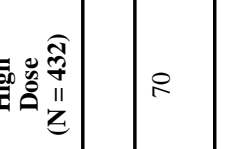

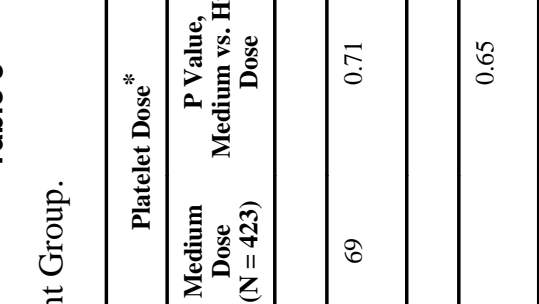

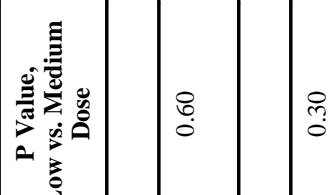

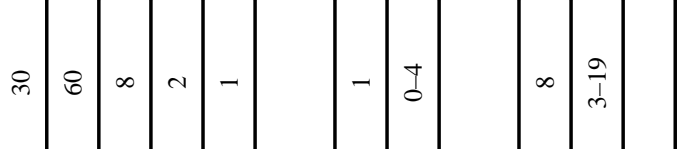
ڤे

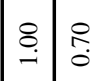
○े

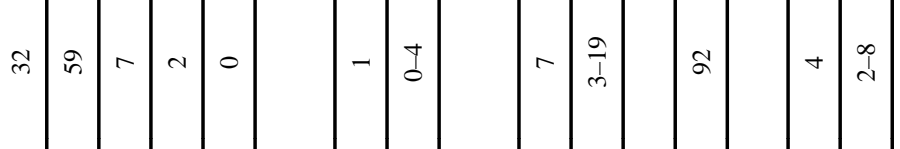

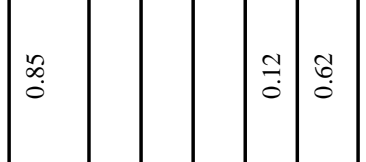

$\overrightarrow{0}$
$\dot{0}$
$\dot{v}$ :

造

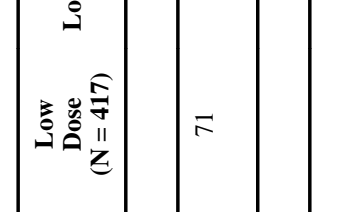

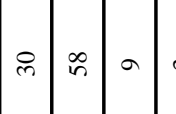

å

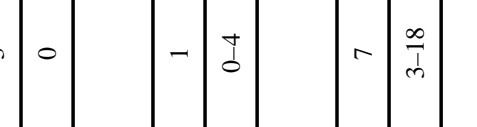

$\approx+\infty \begin{gathered}\infty \\ \vdots \\ c\end{gathered}$

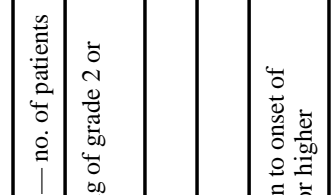

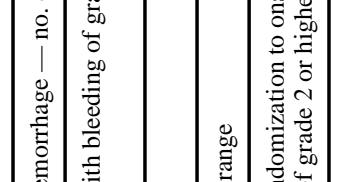

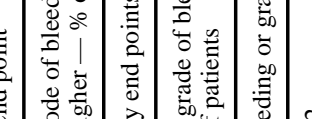




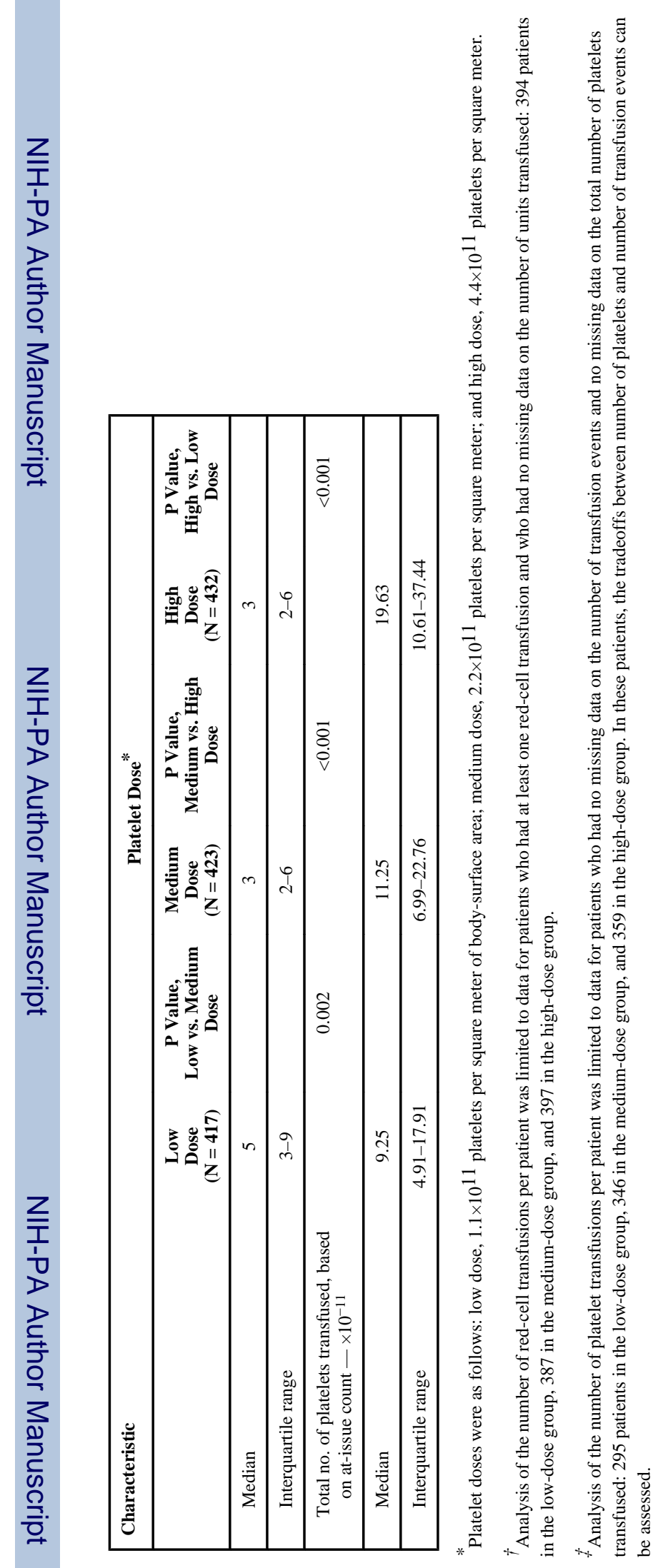

N Engl J Med. Author manuscript; available in PMC 2010 October 7. 


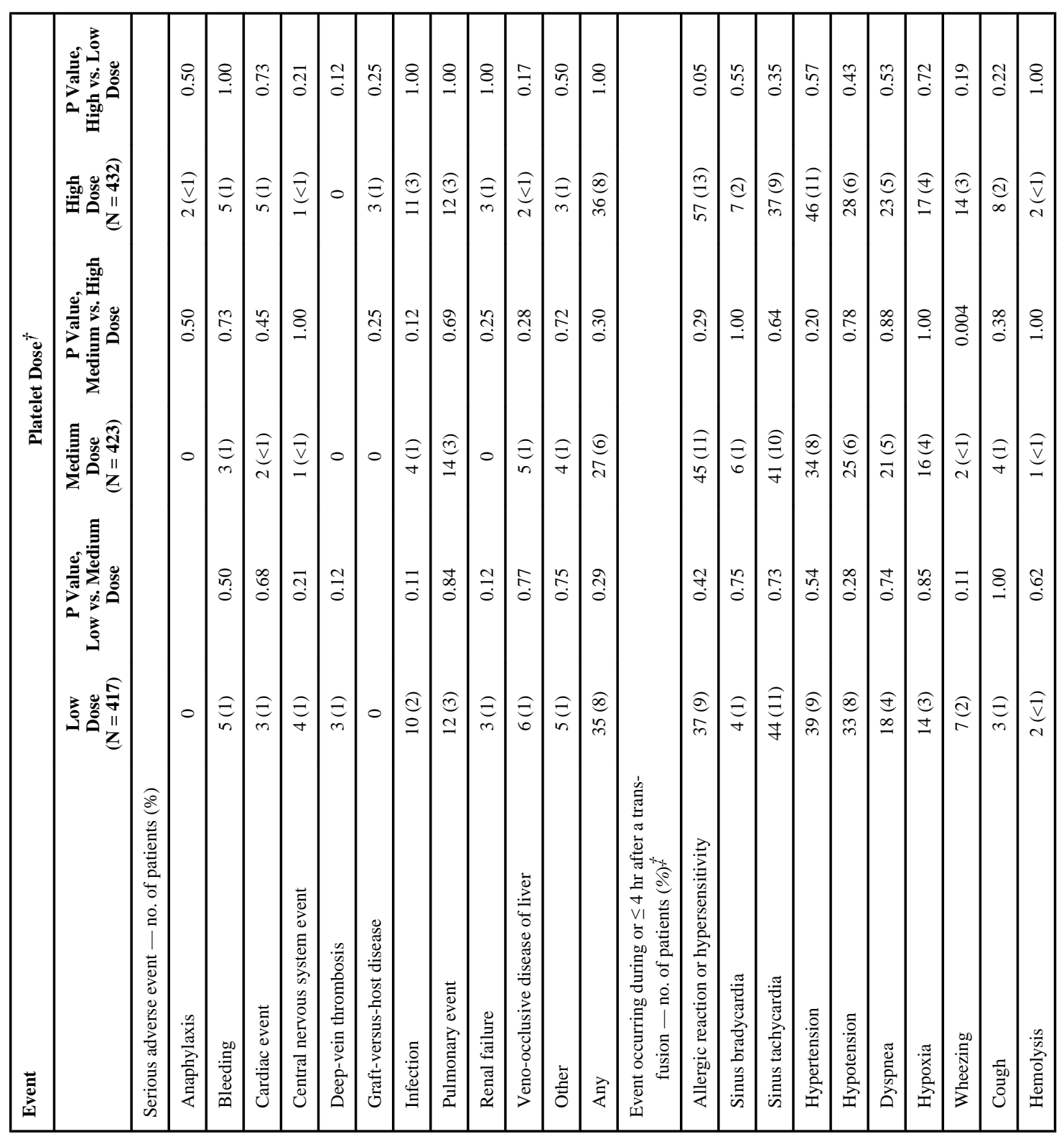

N Engl J Med. Author manuscript; available in PMC 2010 October 7. 


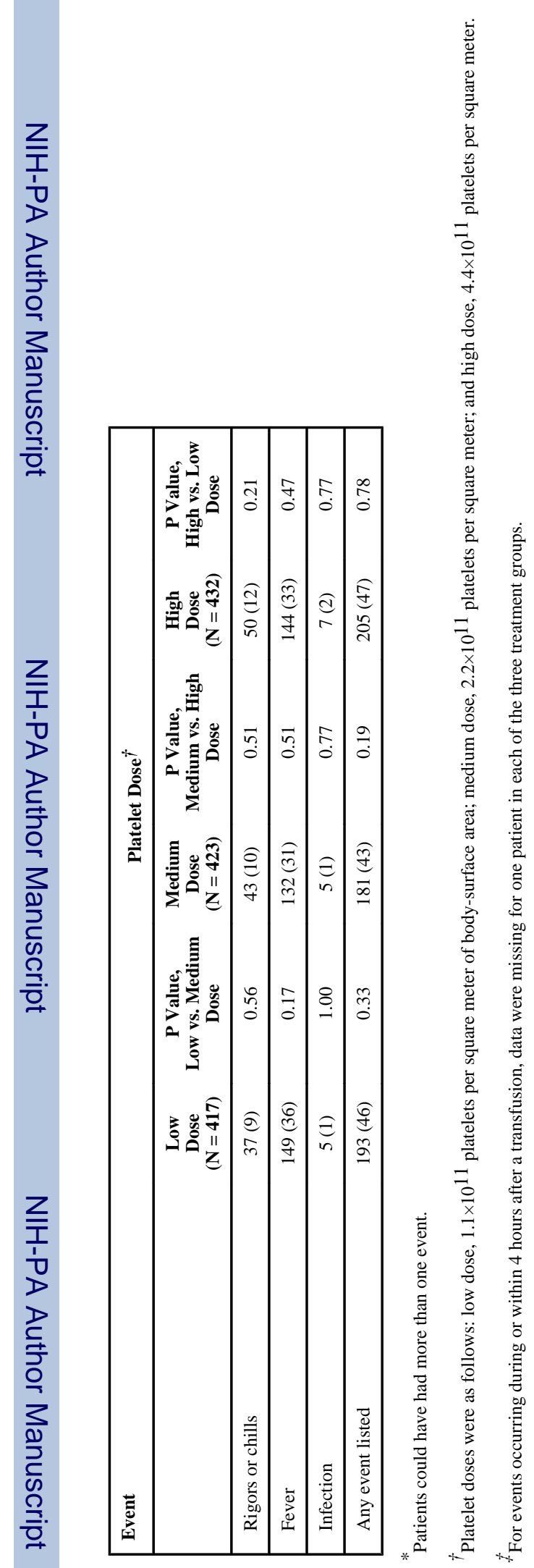

N Engl J Med. Author manuscript; available in PMC 2010 October 7. 